\title{
A Reverse Engineering Approach for Imaging Spinal Cord Architecture - Large Area High-Resolution SEM Imaging.
}

\author{
C. A. Brantner ${ }^{1}$, M. Rasche ${ }^{4}$, K. E. Burcham ${ }^{3}$, J. Klingfus ${ }^{3}$, J. E. Sanabia ${ }^{3}$, C. E. Korman ${ }^{1,2}$, and A. \\ Popratiloff ${ }^{1}$ \\ 1. GW Center for Nanofabrication and Imaging, The George Washington University, Washington, D.C., \\ USA. \\ 2. Department of Electrical and Computer Engineering, The George Washington University, \\ Washington, D.C., USA. \\ 3. International Applications Center, Raith America, Inc., Troy, NY, USA. \\ 4. Raith GmbH, Dortmund, Germany.
}

The CHIPSCANNER, an e-beam lithography instrument, is a scanning electron microscope (SEM) instrument with a laser interferometer stage and electron detectors that has been used for the imaging of integrated circuit boards in the microchip industry. High-resolution, large-area images are created by capturing sequential SEM images and stitching them together for further analyses. The electronics industry uses this technology: to protect against counterfeit products, to verify designs and to recover obsolete designs. Here we show a biological application for the CHIPSCANNER (Raith, Germany). It can be used for imaging tissue samples by generating large area 2D images from many images. We used back-scattered electrons and a cross-section of a rat spinal cord in Epon to show that a biological sample could be imaged in the electron beam lithography instrument. The architecture of a spinal cord is complex and an examination of cellular structures can only be understood by knowledge of the tissue organization as a whole. Thus low magnification images as well as high magnification images are required for understanding. Here, we propose acquisition of high-resolution $2 \mathrm{D}$ registration of adjacent images that includes the entire spinal cord structure.

Image acquisition has challenges to be overcome. The software on many SEM instruments has the capability to collect images in an array and tile them together. There are difficulties in such operations: pixel-to-pixel spacing, limited field of view (FOV), large numbers of images needed to cover large areas of interest, long collection times, stage movement errors, beam position and current drift (causing positional inaccuracy). The CHIPSCANNER overcomes these difficulties with its laser interferometer stage providing accurate sample location allowing for precise stitching together of adjacent images. Responsible for beam deflection and signal recording, the pattern generator efficiently collects and stores images at resolutions up to 50,000 pixels by 50,000 pixels. Figure 1 shows the smooth junction between four images of a microchip that the CHIPSCANNER has imaged and stitched together [1]. Large area images with smooth junctions are important for biology and histology could benefit by visualizing the whole cross section of a tissue.

Spinal cord from a rat was dissected, fixed, and embedded in EPON. Cross-sections were cut and further embedded in resin between Aclar sheets. A resin embedded section was mounted on a stub and imaged in the CHIPSCANNER. Several sample preparation protocols including traditional transmission electron microscope (TEM) and serial blockface protocols were examined to find the set of conditions that allowed for the best preservation of the ultrastructural details as well as the best imaging conditions in the instrument [2]. Figure 2 shows an image from the CHIPSCANNER of the spinal cord. 
We have shown that the CHIPSCANNER can be used to generate high-resolution 2D images from spinal cord samples and is a viable method for obtaining detailed information from large biological samples with an electron beam instrument. TEM is the "gold" standard for resolution and contrast of samples, yet the FOV and area that can be imaged is relatively small compared to the size of the tissue that the cells are organized into. Focused Ion Beam SEM (FIB SEM) is another method to image cells and tissue at high resolution, however it is not capable of large area imaging as can be accomplished in the CHIPSCANNER. There are methods available to image tissues in the field of Histology that scan stained, vibratomed sections on slides using a light microscope and a camera. This scanning and imaging of a slide with a light microscope is similar to the operation of the e-beam CHIPSCANNER.

\section{References.}

[1] J Klingfus, KE Burcham, M Rasche, T Borchert and N Damnik, ISTFA Conference Proceedings (2011), p. 373-376.

[2] T Deerinck, E Bushong, A Thor, M Ellisman, (2010) NCMIR methods for 3D EM: A new protocol for preparation of biological specimens for serial block face scanning electron microscopy. Microscopy: 6-8.

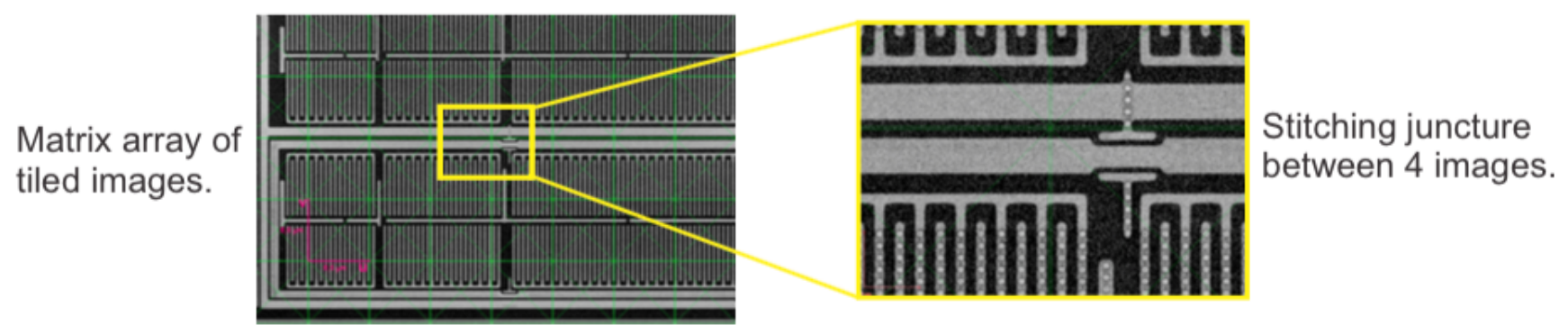

Figure 1. Diagram showing images of a microchip taken with the CHIPSCANNER that have been stitched together. The pink scale bar represents $9 \mu \mathrm{m}$. This system relies on the precision locations of the stage and the e-beam, instead of algorithms in the software for stitching images together to display a large 2D area of the sample.

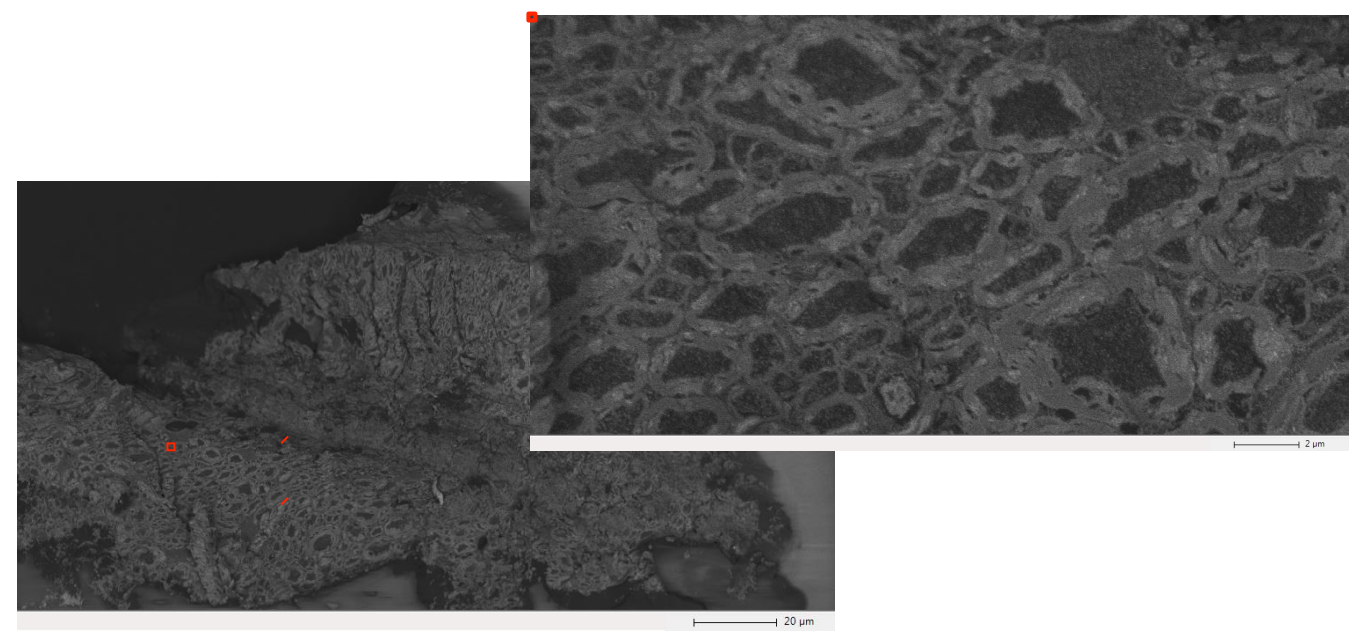

Figure 2. A high resolution image of rat spinal cord taken in the CHIPSCANNER. Left is a 200 $\mu \mathrm{m} \times 100 \mu \mathrm{m}$ area where the image contains 40,000 pixels $\times 20,000$ pixels, corresponding to 1 pixel being $5 \mathrm{~nm} \times 5 \mathrm{~nm}$. The scale bar is $20 \mu \mathrm{m}$. Right is a close-up section from the same image, where the scale bar is $2 \mu \mathrm{m}$. 